\title{
TITLE: EFFICACY OF BRACHIAL PLEXUS BLOCK AFTER ELBOW FRACTURE REPAIR IN CHILDREN
}

AUTHORS: Khalid Chowdary*, FCPS, MD, Kimmo Murto*, FRCPC MD, William Splinter*, FRCPC, $\mathrm{MD}$, Nicholas Barrowman ${ }^{*}, \mathrm{PhD}$.

AFFILATION: * Department of Anesthesiology, Children's Hospital of Eastern Ontario. ${ }^{*}$ Chalmer's Research Group, CHEO Research Institute, 401 Smyth Rd, Ottawa, On K1H 8L1.

INTRODUCTION: Supracondylar fracture of the humerus occurs in 3\% of pediatric trauma cases. Treatment of a grade II and III fracture is closed reduction and percutaneous pinning (1). Postoperative pain increases patient/parent anxiety, delays patient discharge, increases cost of care, and decreases patient/parent satisfaction. Several methods to provide postoperative analgesia include regular interval IV/IM opioids; nurse controlled analgesia (NCA), patient controlled analgesia PCA, and regional analgesia. Opioids are typically used, but are limited by side effects such as respiratory depression, nausea, vomiting, and pruritus. A brachial plexus block (BPB) is an effective mean of providing postoperative analgesia after supracondylar fracture in children $(2,3)$. We performed a retrospective, casecontrol study to evaluate the duration of analgesia provided by brachial plexus block compared to conventional opioid-based analgesia in children after closed reduction and pinning of the supracondylar fracture of the humerus.

METHODS: After institutional ethics board approval, we reviewed cases of children who underwent surgical closed reduction and pinning of supracondylar fracture. Cases done during a period of 46 consecutive months following January 2001 at our institution were reviewed. Patients were excluded if they had an associated congenital developmental, musculoskeletal anomaly or associated traumatic injuries. Patients were divided into two groups based on the analgesic received in the operative period. An intervention group (IG) received a general anesthetic and a brachial plexus block. A Control group (CG) received a general anesthetic with either a combination or single intravenous opioid or ketorolac. Data was collected for the first 24 hour after surgery. The primary outcome measure was time to first dose of opioid after surgery. Undesirable side effects including postoperative vomiting (POV), pruritus, and incidence of compartment syndrome were recorded. Data are presented as proportions or means $\pm \mathrm{SD}$.

RESULTS: Demographic data were similar. Both groups (IG and CG) had 64 patients. Time to first dose of analgesia after surgery in the IG was $9 \pm 3 \mathrm{hr}(\mathrm{p}<0.05)$, and $3 \pm 2 \mathrm{hr}(\mathrm{p}<0.05)$ in CG. The incidence of POV was $19 \%$ (IG) and 32\% (CG). Incidence of pruritus was $6 \%$ (IG), and $10 \%$ (CG). Three patients in each group had swelling of fingers that was secondary to tight fit of the plaster cast around elbow in the first 6 hours of surgery. These were treated with splitting of the plaster cast.

DISCUSSION: It appears that a brachial plexus block provides prolonged pain relief after isolated supracondylar fracture repair in children. In addition it may be associated with fewer side effects as compared to opioids. We suggest a prospective randomized control trial.

\section{REFFERNCES:}

1. Clin Orthop 1987; 219: 174-184.

2. Br J Anaesth 1986; 58: 760-778.

3. Acta Anesth Belg 1981; 4: 301-315. 